\title{
Análisis de la utilización del entorno virtual de aprendizaje por parte de los docentes
}

\section{Analysis of the use of virtual learning environment by teachers}

Lcda. Diana Flor García Calle, MSc.

Universidad Agraria del Ecuador, Ecuador

Ing. Jorge Antonio Ruso León, MSc.

Universidad Agraria del Ecuador, Ecuador

Autor corresponsal:dfgarcia@uagraria.edu.ec,jruso@uagraria.edu.ec

Fecha de recepción: 30 de Agosto de 2017 - Fecha de aceptación: 15 de Abril de 2018

Resumen: La investigación realizada, analiza la utilización del entorno virtual de aprendizaje (EVA) por parte de los docentes de la Facultad de Economía Agrícola en la Universidad Agraria del Ecuador durante el Primer Semestre del año 2016. Para la recopilación de datos se empleó una encuesta a los 41 docentes que son parte de la Facultad y se realizó la revisión de la sesión del EVA a la que cada docente tiene acceso. Esto permitió conocer la utilización de las actividades y recursos del EVA y las opiniones del profesorado sobre su utilidad. Los datos obtenidos muestran que aunque el $78 \%$ de los docentes considera útil el uso del EVA para el proceso de enseñanza/aprendizaje, solo el $12 \%$ indican que el EVA representa un elemento fundamental de este proceso. Además, solo el $10 \%$ de los docentes poseen iniciativa propia al momento de utilizar el EVA y apenas el 7\% de docentes indican que su habilidad para dominar esta herramienta es Excelente. Por otro lado, se confirmó que los profesores ocasionales utilizan con mayor frecuencia el entorno virtual con relación a los de nombramiento. El trabajo realizado identifica la necesidad de formar a los docentes en la utilización adecuada del EVA.

Palabras Claves: tecnología; enseñanza; moodle; plataforma e-learning

\begin{abstract}
The research carried out analyzes the use of the virtual learning environment (EVA) by the teachers of the Faculty of Agricultural Economics at the Agrarian University of Ecuador during the First Semester of 2016. For the data collection, a The 41 teachers who are part of the Faculty and the review of the EVA session to which each teacher has access. This allowed to know the use of the activities and resources of the EVA and the opinions of the teachers about their usefulness. The data show that although $78 \%$ of teachers consider the use of VAS to be useful for the teaching / learning process, only $12 \%$ indicate that VAS represents a fundamental element of this process. In addition, only $10 \%$ of teachers have their own initiative when using EVA and only $7 \%$ of teachers indicate that their ability to master this tool is excellent. In the review of the session for each teacher, it was confirmed that occasional teachers use the virtual environment more frequently in relation to appointment. The work identified identifies the need to train teachers in the proper use of EVA.
\end{abstract}

Key Words: technology; teaching; moodle; e-learning platform 


\section{Introducción}

La sociedad de la información y del conocimiento en la que estamos inmersos nos plantea una serie de retos entre los cuales destacan la necesidad de formar ciudadanos capaces de descodificar, resignificar y transformar la información en conocimiento aplicado (Heargreaves, 2003). En la actualidad, es responsabilidad ineludible de la educación superior asumir estos retos tecnológicos que se plantean a las nuevas generaciones, de manera tal que se desarrollen prácticas educativas que impulsen el acceso y el uso de las Tecnologías de la Información y Comunicación ${ }^{1}$, por parte de los estudiantes y docentes como agentes activos del proceso de enseñanza/aprendizaje.

Según Barkley (2007), "las instituciones educativas deben diseñar metodologías didácticas centradas en el estudiante y en un modelo mediante el cual se desarrolle un proceso de conformación del conocimiento de modo colaborativo". A este respecto, el uso de las TIC's ha tenido, en fechas recientes, una expansión muy grande dentro de las instituciones de educación superior lo que ha llevado a la introducción en alto grado de los Entornos Virtuales de Aprendizaje ${ }^{2}$.

Lévy (2007) indica que las formas sociales, políticas, económicas y culturales propias de la sociedad del siglo XXI han dado lugar al surgimiento de lo que se conoce como la cultura de la sociedad digital. Por otro lado, Bustos y Coll (2010), hacen reflexión acerca de "la capacidad transformadora que las TIC's representan para la educación en la denominada "sociedad del aprendizaje", "sociedad del conocimiento" o "sociedad-red" (Castells, 2001, 2006; Coll y Martí, 2001, citados en Bustos y Coll, 2010). De esta forma, las tecnologías abren las puertas a una nueva manera de acceder, gestionar y construir el conocimiento".

Un hecho trascendental es que la aparición de las nuevas tecnologías ha propiciado a su vez, la aparición de nuevos entornos educativos basados totalmente en las TIC, como los llamados entornos virtuales de aprendizaje. En este sentido, partiendo de lo planteado por Chan (2004), el entorno digital, ha permitido replantear las nuevas formas de aprender, al igual que "los escenarios del espacio para aprender y de las actividades de los estudiantes para acceder, apropiar y procesar información, así como para desarrollar competencias profesionales".

La Universidad Agraria del Ecuador (UAE), es una universidad pública del Ecuador, fundada en 1992 que ofrece formación profesional en carreras de ciencias agrarias, agroindustriales, veterinarias, informáticas y económicas. Asimismo, ofrece cursos de posgrado en especialidades empresariales. La presente investigación pretende realizar un estudio del EVA existente en la Facultad de Economía Agrícola de la UAE con el fin de analizar los usos del mismo por parte de la planta docente de dicha Facultad, para elaborar propuestas de mejora referidas al diseño tecno pedagógico del EVA y detectar necesidades técnicas y pedagógicas del profesorado en relación al uso de dicho entorno. El personal docente de la mencionada facultad

\footnotetext{
${ }^{1}$ TIC

${ }^{2}$ EVA, un entorno virtual de aprendizaje es un espacio educativo alojado en la web, conformado por un conjunto de herramientas informáticas que posibilitan la interacción didáctica (Salinas, 2011).
} 
está integrado por 41 docentes $^{3}: 10$ con nombramiento y 31 ocasionales. Cabe indicar que desde el punto de vista de género hay 17 mujeres y 24 hombres como docentes.

El EVA es desarrollado en la plataforma MOODLE ${ }^{4}$, la cual es un instrumento vital para los docentes, debido a que permite realizar actividades de enseñanza-aprendizaje en el aula, a través de opciones multimedia (Ros, 2008).

La importancia del presente estudio se debe a que las herramientas que presenta el EVA existente en la Facultad de Economía Agrícola de la UAE a disposición de profesores y estudiantes no son explotadas en toda su variedad y potencial con el fin de generar un entorno más colaborativo de aprendizaje y que involucre el uso intensivo de las TIC's. Se pretende caracterizar la utilización del EVA desde el punto de vista de los docentes, como gestores principales de estos sistemas según Gros y Contreras (2006), determinar las causas de la situación que se detecten y plantear las propuestas de mejora que sean procedentes a partir del siguiente objetivo o problema de estudio: Analizar la utilización del entorno virtual de aprendizaje por parte de los docentes de la Facultad de Economía Agrícola en la Universidad Agraria del Ecuador.

\section{Fundamentación teórica}

Los EVA son plataformas de e-learning", Boneu (2007) describe que "las plataformas de e-learning son el software de servidor que se ocupa principalmente de la gestión de usuarios, cursos y de la gestión de servicios de comunicación”. Plantea, asimismo, que hay cuatro características básicas, e imprescindibles, que cualquier plataforma de e-learning debería tener: interactividad, flexibilidad, escalabilidad y estandarización.

La tecnología aplicada a la educación universitaria, mejora la docencia tradicional a través de la Red, no la sustituye. De esta forma es un medio de aprendizaje más dinámico, el cual logra el mejor aprovechamiento de los recursos disponibles (Carabantes et al., 2006). En los últimos años se han dedicado a analizar las posibilidades que estas herramientas pueden tener para la educación. En concreto, estas herramientas presentan una serie de características específicas que sintetizan en las siguientes según Cabero y Llorente (2005):

1. Han sido desarrolladas específicamente para el ámbito educativo.

2. Integran diferentes aplicaciones de internet.

3. No requieren software ni hardware específico por parte del usuario, lo que permite acceder de forma rápida y fácil a través de cualquier navegador.

Expresa Boneu (2007) que otras características generales observables en las plataformas de e-learning son: código abierto, plataforma gratuita, internacionalización, etc. A manera de resumen, se podría decir que el objetivo de las plataformas educativas integra una serie de componentes que son de ayuda para llevar a cabo las acciones formativas a distancia soportadas

\footnotetext{
${ }^{3}$ Datos proporcionados por la Secretaría de la Facultad de Economía Agrícola de la UAE.

${ }^{4}$ Moodle es una aplicación WEB de tipo Ambiente Educativo Virtual, fue creado en 2002 por Martin Dougiamas, quien fue administrador de WebCT en la Universidad Tecnológica de Curtin.

${ }^{5}$ El e-learning consiste en la educación y capacitación a través de Internet. Este tipo de enseñanza online facilita el aprendizaje mediante la utilización de diversas herramientas informáticas.
} 
en la red. Desde un punto de vista técnico, la plataforma que se utilice debe funcionar bajo la filosofía de cliente-servidor y tener una buena interfaz gráfica que permita, de forma muy intuitiva, que los usuarios se puedan desplazar sobre ella (Cabero y Llorente, 2005).

Como indica Suárez Guerrero (2002), los Entornos Virtuales son: "un sistema de acción que basa su particularidad en una intención educativa y en una forma específica para lograrlo a través de recursos infovirtuales". Además, indica que el EVA regula y transforma de forma tecnológica la educación proporcionando formas de actuación externa e interna para el aprendizaje. Hiraldo (2013) plantea que el EVA puede definirse como "el conjunto de medios de interacción sincrónica y asincrónica que, con base en un programa curricular, lleva a cabo el proceso de enseñanza y aprendizaje a través de un sistema de administración de aprendizaje". A este respecto, el EVA da lugar a que exista organización del espacio, disposición y distribución de los recursos didácticos, manejo del tiempo y las interacciones que se dan en el aula.

Por otro lado, algunos autores, hacen referencia de un EVA como un software con accesos restringidos, concebido y diseñado para que las personas que acceden a él desarrollen procesos de incorporación de habilidades y saberes. Es un entorno dinámico, con determinadas condiciones físicas y temporales, que posibilitan y favorecen el aprendizaje. Estos entornos se basan en el principio de aprendizaje colaborativo apoyados con el uso de herramientas multimediales que hacen más agradable un entorno interactivo de construcción de conocimiento. El mismo está diseñado para facilitar al profesor la gestión académica de sus clases y ayudar a los estudiantes en el desarrollo de sus cursos a través de internet. Los EVA en principio surgieron como elementos de soporte a la educación a distancia, pero en la actualidad, también se utilizan como complemento a la educación presencial (Hiraldo, 2013).

Actualmente las investigaciones en el campo de las tecnologías en la educación se inclinan hacia el estudio de los usos o prácticas didácticas que los profesores y alumnos realizan con las TIC (Larraz-Rada et al., 2012). La gestión de un EVA, según Farías y Montoya (2009), a diferencia de un entorno presencial en el aula de clases con la exposición del docente, permite incorporar elementos conceptuales, procedimentales y actitudinales, los mismos que proporcionan una formación en competencias en los estudiantes.

Gros y Contreras (2006), indican que "la gestión de un ambiente de aprendizaje es una tarea básica del profesor" y como plantea Wang et al. (2003), "solo es posible lograr una construcción social del conocimiento en un entorno virtual de enseñanza y aprendizaje si el profesor impulsa la interacción entre los estudiantes". Además, Hiraldo (2013) hace énfasis que es importante que el docente brinde un continuo seguimiento y retroalimentación a aquellas actividades que realiza el estudiante en el EVA. Por otro lado, aunque el docente tenga toda la predisposición pedagógica de interactuar con el estudiante a través de este proceso virtual, es necesario que se cuente también con el apoyo administrativo de la institución.

Por otra parte, Wang et al. (2003) Mencionan que "solo es posible lograr una construcción social del conocimiento en un entorno virtual de enseñanza y aprendizaje si el profesor incentiva la interacción entre los estudiantes de modo que haya un aprendizaje entre pares", en consecuencia "es necesario un seguimiento permanente a las actividades realizadas por ambos actores (docentes y alumnos) ya que permitirá evaluar la correcta utilización de los 
recursos de aprendizajes". Y como lo menciona Cabero et al. (2010) "no es suficiente indicar que profesorado debe tener la formación respectiva para las TIC, sino que también se deben establecer medidas oportunas para su capacitación".

\section{Materiales y métodos}

Se utilizaron métodos cuantitativos mediante la aplicación de encuestas de reactivos cerrados para la determinación de las variables relacionadas con el uso del EVA, excepto la variable de frecuencia semanal de entrada al EVA, en el caso de las variables relacionadas con los datos personales y la variable excluida anteriormente se aplicó el método de recolección directa de la información brindada por el EVA y se procesó en hojas de cálculo para determinar los resultados buscados.

La investigación se realizó en varias fases:

1. Se definieron las variables objeto de estudio y las hipótesis a validar en la investigación.

2. Se consultaron en la Secretaría de la Facultad de Economía Agrícola de la UAE los datos de los docentes relacionados con las variables a verificar.

3. Se realizaron encuestas de reactivos cerrados a la totalidad de los docentes con el fin de indagar sus consideraciones acerca de la utilización del EVA en la Facultad de Economía Agrícola de la UAE. Se tomó como muestra el total de la población objeto del análisis, 41 docentes, debido a que el tamaño de muestra calculado era muy similar a dicha cantidad. Con estas encuestas se pretende conocer la disposición a utilizar esta herramienta, saber si la consideran útil o no, si la utilizan por propia motivación o por cumplir alguna indicación, si se considera que dominan la herramienta o no y si constituye un elemento fundamental de su práctica docente o solo un aspecto más dentro de la misma (Ver Anexo No. 1).

4. Se utilizó una cuenta de administrador del EVA, accediendo a los perfiles y cursos de todos los docentes de la Facultad de Economía Agrícola de la UAE, contabilizando cada una de las actividades $^{6}$ y recursos ${ }^{7}$ utilizados por los mismos durante las 18 semanas de duración del 1er. Semestre del curso 2016 - 2017 para las carreras de Economía Agrícola y Ciencias Económicas.

5. Se tabuló la información anterior y se obtuvieron los resultados necesarios para el análisis de las variables a estudiar.

6. A partir del análisis de los resultados obtenidos se procedió a emitir las conclusiones y recomendaciones correspondientes.

Las variables a estudiar que se determinaron son:

- Variables relacionadas con los datos personales: edad y tipo de contrato del profesor.

- Variables relacionadas con los usos del EVA: Utilidad del EVA para el desarrollo de las asignaturas, frecuencia semanal de entrada al EVA, utilización de actividades/recursos del EVA y opinión del profesorado sobre la utilidad del EVA.

\footnotetext{
${ }^{6}$ Son las herramientas del EVA que posibilitan desarrollar un ambiente de trabajo colaborativo y de interacción entre docentes y estudiantes. De aquí en adelante el término se utilizará de acuerdo a esta definición.

${ }^{7}$ Se definen como los elementos del EVA que permiten la transmisión de información desde el docente hasta el estudiante. De aquí en adelante el término se utilizará de acuerdo con esta definición.
} 
Las hipótesis a validar son las siguientes:

H1: El EVA de la Facultad de Economía Agrícola de la UAE no se utiliza en todo su potencial y efectividad por parte del personal docente.

H2: Las herramientas del EVA enfocadas a la colaboración y la interacción entre docente y estudiante son menos utilizadas, dándose prioridad a aquellas cuya función es solo transmitir información.

H3: Los docentes con contrato ocasional utilizan con mayor intensidad el EVA y son más propensos al uso de las herramientas colaborativas.

H4: La edad de los docentes determina el uso del EVA como una herramienta principal de su práctica docente, fomentando el uso de la misma como un espacio de aprendizaje colaborativo y de interacción con los estudiantes.

\section{Resultados}

Los resultados obtenidos sobre el análisis de las variables: edad, tipo de contrato del profesor, frecuencia semanal de entrada al EVA y utilización de actividades/recursos del EVA, se calcularon mediante el procesamiento en hojas de cálculo de los datos recolectados directamente de los perfiles de cada docente en el EVA de la Facultad de Economía Agrícola de la UAE y se resumen a continuación:

1. Se determinaron cincos rangos de edades, para conocer la cantidad de actividades y recursos que suben al EVA. En los docentes menores de 30 años, el $9.76 \%$ del total de docentes, suben al EVA el 34.9\% de las actividades y recursos. El 53.66\% de docentes están comprendidos en las edades de 31 a 40 años y cuelgan el $21.6 \%$ de las actividades y recursos. El $14.63 \%$ del profesorado, se encuentran entre 41 y 50 años y colocan el $23.5 \%$. El $21.95 \%$ de los docentes están en los rangos de 51 a 60 años y mayores a 60, ambos grupos suben en conjunto al EVA el $20 \%$ del total de recursos y actividades (Ver Figura No. 1).

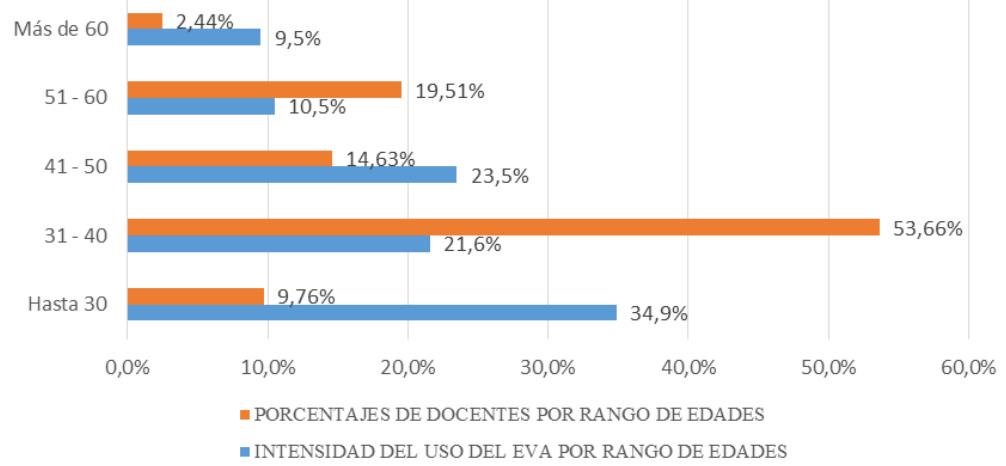

Figura No. 1: Intensidad del uso del EVA por rangos de edad en los Docentes Fuente: Elaboración propia a partir de datos de la encuesta

2. Teniendo en cuenta que el total de semanas de un semestre es 18 y haciendo un contraste entre los tipos de contratos de los profesores se determinó que el 76\% de los docentes son de contrato ocasional y acceden al EVA el $50.72 \%$ del total de las semanas del semestre, lo que 
significa que acceden como promedio 9.12 semanas; mientras que los docentes con nombramiento, el 24\%, utilizan el EVA durante una media de 4.3 semanas, es decir, un $23.89 \%$ del total de las semanas del semestre (Ver Figura No. 2).

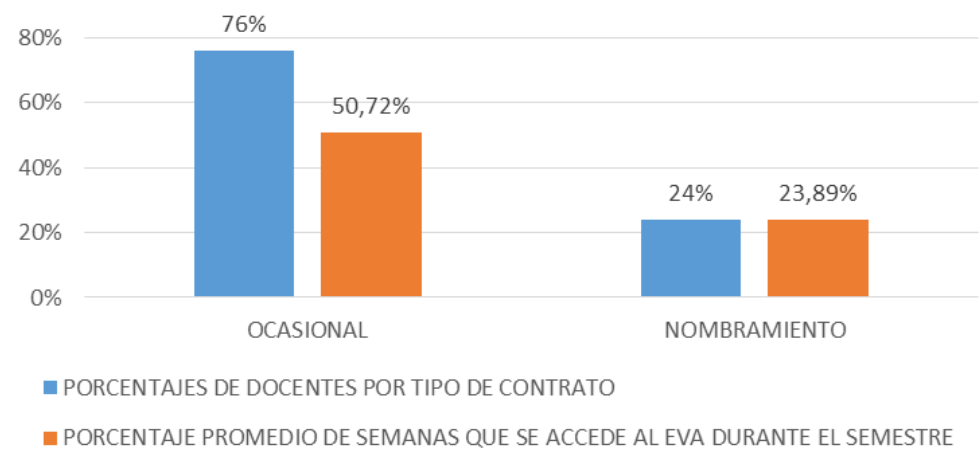

Figura No. 2: Porcentaje de acceso al EVA durante el semestre según tipo de contrato de los docentes

Fuente: Elaboración propia a partir de datos de la encuesta

3. Se constató que los docentes con nombramiento, aunque usan con menor intensidad el EVA, aplican las herramientas colaborativas y de interacción con mayor frecuencia que los docentes ocasionales. Esto puede deberse a dos factores, a saber: (1) los docentes de nombramiento pueden tener mayor experiencia y/o conocimientos en la utilización del EVA que aquellos que son ocasionales y (2) los ocasionales con el fin de cumplir con las exigencias de uso del EVA por parte de los directivos solo cuelgan herramientas de transmisión de información, las cuales tienen un impacto limitado desde el punto de vista de un proceso de enseñanza/aprendizaje interactivo lo que va en detrimento del uso de herramientas colaborativas (Ver Tablas No. 1 y 2).

Tabla No. 1: Porcentaje de uso de actividades y recursos por parte de los docentes con nombramiento. Los ítems sombreados corresponden a las actividades, el resto son recursos.

\begin{tabular}{lr}
\hline Uso de actividades y recursos \\
\hline Archivo & $56,52 \%$ \\
Tarea & $39,13 \%$ \\
Cuestionario & $1,45 \%$ \\
Wiki & $1,45 \%$ \\
Libro & $1,45 \%$ \\
URL & $0,00 \%$ \\
Carpeta & $0,00 \%$ \\
Foro & $0,00 \%$ \\
Taller & $0,00 \%$ \\
Página & $0,00 \%$ \\
Chat & $0,00 \%$ \\
Consulta & $0,00 \%$ \\
Encuesta & $0,00 \%$ \\
Glosario & $0,00 \%$ \\
Mensajes & $0,00 \%$ \\
Asistencia & $0,00 \%$ \\
Etiqueta & $0,00 \%$ \\
\hline
\end{tabular}

Fuente: Elaboración propia de los datos recopilados del Aula Virtual de la UAE

Tabla No. 2: Porcentaje de uso de actividades y recursos por parte de los docentes ocasionales. Los ítems sombreados corresponden a las actividades, el resto son recursos. 


\begin{tabular}{lr}
\hline Uso de actividades y recursos \\
\hline Archivo & $56,96 \%$ \\
Tarea & $23,70 \%$ \\
URL & $4,35 \%$ \\
Mensajes & $4,13 \%$ \\
Carpeta & $2,61 \%$ \\
Foro & $2,17 \%$ \\
Taller & $1,74 \%$ \\
Asistencia & $1,09 \%$ \\
Cuestionario & $0,87 \%$ \\
Wiki & $0,43 \%$ \\
Página & $0,43 \%$ \\
Etiqueta & $0,42 \%$ \\
Libro & $0,22 \%$ \\
Chat & $0,22 \%$ \\
Consulta & $0,22 \%$ \\
Encuesta & $0,22 \%$ \\
Glosario & $0,22 \%$
\end{tabular}

Fuente: Elaboración propia de los datos recopilados del Aula Virtual de la UAE

4. Se comprobó que la frecuencia promedio semanal de entrada al EVA por parte de los docentes, sin distinción de tipo de contrato, es del $44.17 \%$ del total de semanas del semestre académico, lo que representa 7.95 semanas, esto significa que un docente accede a interactuar con el EVA, como media, poco menos de 1 vez cada 2 semanas.

5. En cuanto al análisis del uso que se hace de las actividades y recursos del EVA por parte de todos los docentes, se observó que los cincos primeros puestos son ocupados por 4 recursos y 1 actividad, no obstante, un significativo porcentaje, el $82.61 \%$, del uso de recursos y actividades queda en los dos primeros puestos: el recurso "Archivo" con un $56.90 \%$ y la actividad "Tarea" con un 25.71\%. Estos resultados evidencian claramente dos aspectos: (1) el uso que se hace de las variadas herramientas que el EVA pone a disposición de docentes y estudiantes es muy limitado y (2) el mayor uso que se da al EVA está en función solamente de la transmisión de información, lo cual no es su principal propósito, quedando relegadas en su utilización aquellas herramientas que pueden generar un proceso de enseñanza/aprendizaje que promueva un entorno colaborativo y de interacción entre profesores y alumnos (Ver Tabla No. 3).

Tabla No. 3: Porcentaje de uso de actividades y recursos por parte de todos los docentes. Los ítems sombreados corresponden a las actividades, el resto son recursos. 


\begin{tabular}{lr}
\hline Uso de actividades y recursos \\
\hline Archivo & $56,90 \%$ \\
Tarea & $25,71 \%$ \\
URL & $3,78 \%$ \\
Mensajes & $3,59 \%$ \\
Carpeta & $2,27 \%$ \\
Foro & $1,89 \%$ \\
Taller & $1,51 \%$ \\
Asistencia & $0,95 \%$ \\
Cuestionario & $0,95 \%$ \\
Wiki & $0,57 \%$ \\
Página & $0,38 \%$ \\
Etiqueta & $0,37 \%$ \\
Libro & $0,37 \%$ \\
Chat & $0,19 \%$ \\
Consulta & $0,19 \%$ \\
Encuesta & $0,19 \%$ \\
Glosario & $0,19 \%$ \\
\hline
\end{tabular}

Fuente: Elaboración propia de los datos recopilados en el Aula Virtual de la UAE

Con estos resultados se valida la hipótesis $\mathbf{H 1}$ por cuanto se constata que el EVA de la Facultad de Economía Agrícola de la UAE no es utilizado en todo su potencial y efectividad. Asimismo, se evidencia el escaso uso que se hace del EVA por una importante parte del profesorado.

También se confirma la hipótesis $\mathbf{H 2}$ pues se demuestra que esta herramienta es utilizada prioritariamente para la transmisión de información y no para incentivar la colaboración e interacción entre docentes y estudiantes.

Con respecto a la hipótesis H3, ésta se corrobora en parte pues queda establecido que son los docentes ocasionales quienes usan con mayor intensidad el EVA, colgando, asimismo, más del doble de actividades/recursos que los docentes con nombramiento, sin embargo, se detecta que los docentes con nombramiento son quienes más utilizan las herramientas colaborativas e interactivas, por ello la $\mathbf{H 3}$ no se valida en lo referente a este último aspecto.

En cuanto a la hipótesis $\mathbf{H 4}$ se comprueba que, de manera general, no existe una correlación directa entre edad e intensidad de uso del EVA, es decir, entre más jóvenes son los docentes, con mayor intensidad utilizan el EVA y viceversa, sino que, solo se cumple en el rango de los menores de 30 años, no siendo así para el resto de los rangos, por lo que se invalida la presunción hecha.

Para obtener los resultados del análisis de las variables: utilidad del EVA para el desarrollo de las asignaturas y las opiniones del profesorado sobre la utilidad del EVA, se procesaron las 41 encuestas realizadas lo cual arrojó la siguiente información:

- De los 41 docentes, 13 de ellos, lo que corresponde al 32\% indicó sentirse motivado a utilizar el EVA, mientras que los 28 docentes restantes, es decir el 68\%, respondió no sentirse motivado.

- $\quad$ El 78\% de los docentes, 32 personas, expresó que considera útil el uso del EVA para el proceso de enseñanza/aprendizaje, por su parte, los 9 profesores restantes, el $22 \%$, plantearon que el EVA no tiene una utilidad significativa para la enseñanza. 
- $\quad$ El 7\% de docentes, que corresponden a 3 profesores, indicaron que sus pericias para el manejo del EVA son "Excelentes"; otro 12\%, 5 docentes, expresaron tener "Muy buenas" destrezas con el EVA; el 22\% de docentes, 9 profesores, dijo que posee una experticia en la gestión del EVA que califica como "Buena"; por otro lado, el 46\% de los encuestados, 19 profesores, plantearon que su nivel es "Regular" y, finalmente, el 12\% de docentes, representado por 5 docentes, declararon no tener destreza alguna en el manejo del EVA por lo que marcaron sus habilidades como "Malas".

- El 12\% de los docentes, 5 personas, consideran que el Entorno Virtual de Aprendizaje representa un elemento fundamental en el proceso de enseñanza/aprendizaje que desarrolla; y el 88\%, es decir 36 docentes, expresaron que NO es un elemento fundamental.

- Finalmente, el 10\% de los docentes, es decir 4 profesores, indican que poseen iniciativa propia al momento de utilizar el EVA; mientras que el 90\%, 37 docentes, señalaron que utilizan el EVA para cumplir con una disposición de las autoridades de la Facultad de Economía Agrícola.

Del análisis de las encuestas podemos determinar los resultados a que se llega para las variables objeto de estudio por este método y que antes se mencionan:

1. En cuanto a la utilidad del EVA para el desarrollo de las asignaturas y las opiniones del profesorado, se concluye que, aunque la mayoría lo considera útil no lo aplica o solo lo hace de manera muy superficial, pues no se sienten motivados a utilizarlo, en lo cual inciden significativamente las pocas o nulas habilidades para utilizarlo que expresa la mayor parte de la planta docente. Además, en los casos de docentes que lo utilizan con mayor frecuencia y/o habilidad se detecta que el uso que se da al EVA es más como simple herramienta de transmisión de información que como entorno colaborativo e interactivo para el proceso de enseñanza/aprendizaje.

2. Por otra parte, la mayoría de docentes expresan que utilizan el EVA, no por propia iniciativa, sino por cumplir las directrices de la Facultad de Economía Agrícola que exigen la utilización de esta herramienta, esto corrobora los resultados obtenidos en la pregunta 4 del cuestionario, de la que se concluye que el profesorado, en su mayor parte, no considera el uso del EVA como elemento fundamental del proceso educativo, pues al no existir motivación propia no es posible lograr que el docente incorpore conscientemente el EVA en su práctica diaria, en lo cual influye, naturalmente, el bajo grado de pericia en el manejo de la herramienta y el escaso conocimiento de su potencial, lo que anteriormente ya se comentó.

\section{Discusión}

Según Salinas (2011) "La utilización de los EVA debe promover un modelo de enseñanza centrado en el alumno pues son diseñados con ese objetivo, de manera que se utilicen las herramientas tecnológicas que los componen y las estrategias de aprendizaje que pueden proponerse a partir de ellas", sin embargo, los resultados del presente trabajo indican, que por el contrario, el modelo de enseñanza que se aplica en la Facultad de Economía Agrícola de la UAE se centra en el docente pues el trabajo con el EVA se focaliza en la transmisión de información desde el profesor, dicho modelo se limita a la publicación de materiales y la propuesta de 
actividades para la reproducción de esa información, sin mayores procesos de reelaboración personal por parte del alumno. Estos resultados son coincidentes con los obtenidos por LarrazRada et al. (2012).

El mismo resultado anterior se obtiene al analizar otra de las posibilidades de los EVA planteadas por Salinas (2011) y que es la referida a la de "proponer nuevas estrategias metodológicas a partir de la utilización de herramientas tales como los foros, el chat, wiki, la confección de glosarios y otras". En el caso de estudio, se aprecia que son los recursos, es decir, las herramientas de transmisión de información, las que son utilizadas preferentemente por el profesorado, corroborando que el modelo de utilización del EVA está centrado en el docente.

\section{Conclusiones}

En cuanto a la posibilidad de utilizar nuevos recursos didácticos, encontramos la misma situación antes descrita por cuanto que al ser el docente el agente promotor que debe incentivar y configurar el potencial formativo de estas tecnologías, éste debe poseer la capacitación adecuada para desarrollar dicho potencial y ser capaz de generar un modelo de utilización del EVA centrado en el estudiante, no obstante, lo encontrado en la presente investigación demuestra el bajo nivel del dominio del docente siquiera para interactuar con las herramientas más básicas, así como que tampoco existe la motivación consciente para utilizarlo como una herramienta central de la actividad formativa; lo que tiene su origen, precisamente, en que no se conoce suficientemente el manejo de la herramienta y sus potencialidades.

Los docentes ocasionales utilizan con más intensidad el EVA; sin embargo, lo hacen, fundamentalmente, por cumplir una indicación institucional. Los docentes con nombramiento al sentirse con menor presión acceden con menor frecuencia al EVA, sin embargo, utilizan con mayor intensidad las herramientas colaborativas. No necesariamente los docentes más jóvenes, utilizan más el EVA, sino que, falencias tales como el poco conocimiento y manejo de esta herramienta, provocan que gran cantidad de docentes entre 30 y 40 años utilicen menos las herramientas que un grupo menor de entre 40 y 50 años de edad.

A partir de estas conclusiones pueden emitirse las recomendaciones que se relacionan seguidamente:

1. Establecer un sistema de diagnóstico del uso del EVA por cada docente y diseñar las necesidades formativas y técnicas del profesorado.

2. Desarrollar un curso de manejo e interacción con el EVA con los estudiantes de nuevo ingreso a la universidad al inicio de cada semestre.

3. Al comienzo de cada curso actualizar y recapacitar a los docentes sobre el uso del EVA y/o nuevas herramientas o técnicas pedagógicas o de gestión del mismo que se hayan mejorado o agregado. En este caso es prioritario incluir a los nuevos docentes.

4. Exigir a los docentes que incluyan dentro del $\mathrm{PEA}^{8}$ correspondiente a las asignaturas que imparten actividades suficientes y variadas que impliquen explotar el EVA como un recurso frecuente de colaboración e intercambio con los estudiantes.

5. Establecer un manual de consulta docente para detallar los usos pedagógicos del EVA.

\footnotetext{
${ }^{8}$ Programa de enseñanza de la asignatura.
} 
6. Redactar una guía dirigida a los estudiantes sobre los usos del EVA en la UAE.

\section{Bibliografía}

Barkley, E. F. (2007). Técnicas de aprendizaje colaborativo. Manual para el profesorado universitario. Madrid: Ediciones Morata.

Boneu, J. M. (2007). Plataformas abiertas de e-learning para el soporte de contenidos educativos abiertos. Obtenido de Revista de universidad y sociedad del conocimiento: http://www.uoc.edu/rusc/4/1/dt/esp/boneu.pdf

Bustos, A., \& Coll, C. (2010). Los entornos virtuales como espacios de enseñanza y aprendizaje: una perspectiva psicoeducativa para su caracterización y análisis. Revista Mexicana de Investigación Educativa, 163-184.

Cabero, J., \& Llorente, C. (2005). Las plataformas virtuales en el ámbito de la teleformación. Obtenido de tecnologiaedu.us.es: http://tecnologiaedu.us.es/cuestionario/bibliovir/plataformas_virtuales_teleformacion_20 05.pdf

Cabero, J., Barroso, J., \& Llorente, M. (2010). El diseño de Entornos Personales de Aprendizaje y la formación de profesores en TIC. Obtenido de Digital Education Review: http://greav.ub.edu/der

Carabantes, D., Carrasco, A., \& Alves, J. (23 de 10 de 2006). La Innovación a través de los entoprnos virtuales de enseñanza y aprendizaje. Obtenido de http://www.biblioteca.org.ar/libros/142137.pdf

Chan Núñez, M. (11 de 2004). Tendencias en el diseño educativo para entornos de aprendizaje digitales. Obtenido de Revista Digital Universitaria, Núm 10, Vol. 5: http://www.revista.unam.mx/vol.5/num10/art67/int67.htm

Farías Martínez, G. M., \& Montoya del Corte, J. (2009). Gestión de un entorno virtual de aprendizaje para el desarrollo de competencias profesionales interculturales: una experiencia de educación superior entre México y España. Obtenido de Universidad de Cantabria: http://repositorio.unican.es/xmlui/handle/10902/6327

Gros, B., \& Contreras, D. (2006). La alfabetización digital y el desarrollo de competencias ciudadanas. Obtenido de Revista Iberoamericana de Educación: http://rieoei.org/rie42a06.htm

Heargreaves, A. (2003). Enseñar en la sociedad del conocimiento. Barcelona: Ediciones Octaedro.

Hiraldo, R. (2013). Uso de los entornos virtuales de aprendizaje en la educación a distancia. EDUTEC, 3.

Larraz-Rada, V., Sánchez i Valero, J.-A., Casalprim-Ramonet, M., \& Saz-Peñamaría, A. (2012). Análisis del uso didáctico del entorno virtual de aprendizaje de la Universitat d' Andorra 
- Obtenido de Universitat d' Andorra: http://www.uda.ad/wpcontent/uploads/2010/01/siie_2012_larraz_casalprim_sanchez_saz.pdf

Lévy, P. (2007). Cibercultura. La cultura de la sociedad digital. México: Anthropos.

Ros, I. (2008). Moodle, la plataforma para la enseñanza y organización escolar. Obtenido de Ikastorratza, e- Revista: http://www.ehu.es/ikastorratza/2_alea/moodle.pdf

Salinas, M. I. (2011). Entornos virtuales de aprendizaje en la escuela: tipos, modelo didáctico y rol del docente. Obtenido de http://www.uca.edu.ar/uca/common/grupo82/files/educacionEVA-en-la-escuela_web-Depto.pdf

Suárez Guerrero, C. (2002). Los entornos virtuales de aprendizaje como instrumentos de mediación. Salamanca: Ediciones Universidad de Salamanca.

Wang, M., Sierra, C., \& Folger, T. (2003). Building a dynamic online learning community among adult learners. Obtenido de Educational Media International: http://www.tandfonline.com/doi/abs/10.1080/0952398032000092116 


\section{Anexo No. 1}

\section{Encuesta realizada a docentes de la Facultad de Economía Agrícola de la Universidad Agraria del Ecuador}

Encuesta a Docentes: Utilización del Entorno Virtual de Aprendizaje por parte de los Docentes de la Facultad de Economía Agrícola de la UAE.

Estimado docente: Como parte de una investigación que se lleva a cabo para conocer el uso del EVA (Entorno Virtual de Aprendizaje) en la Facultad de Economía Agrícola de la UAE, se solicita llene la presente encuesta, recuerde que es anónima.

Cuestionario: A cada pregunta, seleccione con una " $\mathrm{X}$ " la opción que corresponda o que más se ajuste a Ud.

1. ¿Usted se siente motivado a utilizar el Entorno Virtual de Aprendizaje que pone la Facultad de Economía Agrícola a su disposición?

SI

NO

2. ¿Considera útil el empleo del Entorno Virtual de Aprendizaje para el proceso de enseñanzaaprendizaje?

SI

NO

3. ¿Cómo considera sus habilidades para dominar la potencialidad del Entorno Virtual de Aprendizaje?

EXCELENTE

MUY BUENA

BUENA

REGULAR

MALA

4. ¿Considera que el Entorno Virtual de Aprendizaje representa un elemento fundamental en el proceso de enseñanza-aprendizaje que usted desarrolla?

SI

NO

5. Motivo por el cual utiliza el Entorno Virtual de Aprendizaje.

Iniciativa propia

Obligación en cumplir con la indicación de utilizar el EVA 